\title{
Verschwörungstheorien und Wahninhalte
}

\author{
Hans-Ludwig Kröber ${ }^{1}$ \\ Online publiziert: 10. Juni 2020 \\ (c) Springer-Verlag GmbH Deutschland, ein Teil von Springer Nature 2020
}

\section{Conspiracy theories and delusional contents}

Bereits vor Pfingsten begaben sich die Erleuchteten auf die Straße und gesellten sich zu den Menschen, die gegen Einschränkungen aufgrund der COVID-19-Pandemie demonstrierten. Einige Tausend Bürger protestierten gegen Kita- und Schulschließungen oder Gottesdienstverbote oder Besuchsverbote im Altenheim oder Reisebeschränkungen. Diese, so las man, seien ,instrumentalisiert“ worden, von Rechtsextremisten, pegidophilen Wutbürgern, Linksextremisten und vor allem „Verschwörungstheoretikern“. Die Medien konzentrierten sich auf prominente Vertreter abstruser Theorien, wie den Sänger Xavier Naidoo oder den Autor veganer Kochbücher Attila Hildmann, sowie die vielen anderen Sekten, die mit von der Partie sind, Impfgegner, Chemtrail-Aluhutträger, Reichsbürger und Untergangspropheten. Es gibt sie schon seit Jahrzehnten, aber jetzt sind sie aus dem Netz gekrochen, und alle Sekten gleichzeitig.

Nun machen allerdings einige dieser Herren den Eindruck, dass sie nicht nur abstruses Zeug reden, sondern tatsächlich psychotisch dekompensiert sind. Für den Psychiater ergibt sich die Frage, woran man festmachen kann, ob eine Person verstiegenen, abseitigen Theorien anhängt und sich im Kreis von Gesinnungsgenossen wohlfühlt, oder ob sie wahnkrank ist. Dann tut sich die nächste Frage auf, ob dieser „Verschwörungstheoretiker" unter einer anhaltenden wahnhaften Störung leidet oder unter einer paranoiden Schizophrenie. Witter (1972, S. 471) hat einst eine Steigerungsreihe benannt, nämlich Irrtum - Vorurteil - überwertige Idee - Wahneinfall/Wahnvorstellung als Stufen einer paranoiden Entwicklung, von welcher der schizophrene Wahn abzugrenzen ist.

Irrtum bleibt wesentlich auf der kognitiven Ebene und wird bei sachlicher Widerlegung korrigiert. Das Vorurteil

Prof. Dr. Hans-Ludwig Kröber

mail@hlkroeber.de

1 Zentrum für Forensisch-Psychiatrische Begutachtung (ZFPB), Schloßstraße 50, 12165 Berlin, Deutschland ist bereits deutlich emotional unterlegt und bleibt lebendig, auch wenn es reichlich Gegenbeweise gibt - die dann als Einzelfälle gelten, die das Vorurteil nicht widerlegen.

Wenn ein Vorurteil nicht einen umschriebenen Bereich abdeckt, sondern das ganze Denken und Fühlen zu lenken beginnt, sprechen wir von einer überwertigen Idee. Witter verweist darauf, dass gerade auch die öffentlichen Medien „,namentlich in Zeiten einer allgemein erhöhten Affektanspannung und Affekterregung "für die Verbreitung von Vorurteilen und überwertigen Ideen sorgen - es gab 1972 noch kein Internet. Im Rahmen einer paranoiden Entwicklung kann sich daraus ein stabiler ein Liebeswahn, Eifersuchtswahn, Querulantenwahn oder eben auch Verfolgungswahn entwickeln und in einem Wahnsystem verfestigen, in dem die diversen wahnhaften Überzeugungen sich wechselseitig beweisen.

Viele „Verschwörungstheoretiker" tragen ihre überwertige Idee missionarisch zu Markte. Nehmen wir die Impfgegner. Es gibt sie so lange, wie es Impfungen gibt. In meiner Kindheit zogen Impfgegner gegen die Polioschluckimpfung zu Felde; der von Sabin entwickelte Lebendimpfstoff sei hochgefährlich. Auch heute noch dürfte die Mehrheit der Impfgegner/Impfgegnerinnen medizinischen Ideologien anhängen, wonach Impfungen erstens unnötig seien und zweitens viel zu oft schwere Krankheiten verursachen. Dass sie trotzdem staatlich vorgeschrieben sind, diene allein der Pharmaindustrie.

Die „Verschwörung“ bei vielen Verschwörungstheorien reduziert sich meistens darauf, dass irgendein für gefährlich gehaltenes Phänomen entweder dem Großkapital dient, gar dem jüdischen, oder dem Staat bzw. den herrschenden Eliten oder bestimmten Kombinationen von Kapital und mächtigen Personengruppen. Diese kausale Ableitung (Kapital und Obrigkeit) wird nicht nur von Jusos, sondern von vielen Bürgern für plausibel gehalten, nicht hingegen die Theorie des SPD-Politikers Wodarg, die Schweinegrippe sei eine Erfindung der Pharmaindustrie gewesen, und mit SARS-CoV-2 sei es nicht anders. Wodarg war als Amtsarzt 
übrigens Vorgesetzter des Hochstaplers Gerd Postel gewesen, durchschaut also nicht jede Täuschung.

Wer „normale“ Impfgegner erlebt hat, wird nicht auf die Idee kommen, sie für psychotisch zu halten, auch wenn er sich fragt, woher die hohe emotionale Besetzung des Themas bei diesen Menschen rührt. Das ist so ähnlich wie bei den militanten Atheisten, die ungebeten für den Atheismus missionieren und offenbar ein großes Problem mit Gott haben, den es doch gar nicht gibt. Das ist interessant, aber nicht psychotisch.

Nun aber war just in der Woche der Demonstrationen ein Psychiatriepatient Herr A. zu begutachten, 47 Jahre alt, Nichtraucher und Abstinenzler, der vor 13 Jahren die deutsche Regierung angezeigt hat, wegen krimineller Machenschaften, so der Teilnahme an Geheimkonferenzen von Politik, Militär und Wirtschaft, wegen Foltern der Bevölkerung und auch seiner eigenen Person. Bei einer Begutachtung 2017 hatte er erklärt, die deutsche Regierung habe seit 1995 310 Mrd. für Chemtrail ausgegeben, um die Bevölkerung zu vergiften; sie habe das 2015 selbst zugegeben. Auf die Frage: warum? - Das solle man Merkel fragen. Die BASF suche Chemtrail-Piloten, es gebe genug Beweise. Jetzt werde das durch Impfungen durchgeführt, weil das billiger sei.

Zudem und vor allem, so Herr A., werde er mit Mikrowellen gefoltert. Das Fraunhofer-Institut in Bonn habe ein Waffensystem mit Mikrowellen erforscht. Man lese „die Resonanz" von Menschen mit Computer und Radar aus. Dann werde diese Frequenz in Mikrowellen eingekoppelt und bestrahlt. Das sei alles im Internet von Wissenschaftlern bewiesen. Die Strahlenfolter habe beabsichtigt und erreicht, dass seine persönlichen Beziehungen (zu Frauen) zu Bruch gegangen seien, und er habe ihretwegen Krebsknoten in der Brust. Aber Donald Trump werde der deutschen Regierung Humanität beibringen, jetzt komme frischer Wind aus den USA. Und wenn Merkel gestürzt sei, werde alles ans Tageslicht kommen.

Dieser Mann lebte seit Jahren arbeitslos und allein, war sehr viel im Internet unterwegs und traf sich mit Gleichgesinnten. Er schrieb angeblich 11.000 Haushalte sowie verschiedene Ärzte an, um auf die Folter aufmerksam zu machen, und plakatierte schließlich gegen die Bestrahlungsfolter, um auf die Gräueltaten dieser „Nazi-Abschaum“Regierung aufmerksam zu machen. Weil er, um Polizisten am Eindringen in seine Wohnung zu hindern, reichlich Benzin an seiner Wohnungstür vergossen und angezündet hatte, wurde er begutachtet. Er war völlig geordnet, frei von formalen Denkstörungen, hatte adäquate soziale Umgangsformen, wirkte im Kontakt nicht schrullig oder merkwürdig, war aber sehr engagiert beim Vortragen seiner Erkenntnisse. Von Gegenvorstellungen war er nicht zu erreichen; er nahm sie gar nicht zur Kenntnis.
Der Kollege diagnostizierte eine wahnhafte Störung (ICD-10 F22.0). Das überzeugt, auch wenn die Informationslage eingeschränkt war und Herr A. sehr wenig über seine Lebensgeschichte und sein eigenes Erleben preisgegeben hatte. Schon als Zeitsoldat war er einmal aufgefallen, weil er über Nachstellungen von Vorgesetzten klagte, und dass die Kameraden hinter seinem Rücken über ihn lachen; man hatte im Bundeswehrkrankenhaus eine Psychose verneint. Nach sechs Jahren Bundeswehr fand er nicht ins Leben, stieg aus, widmete sich esoterischen Weisheitslehren und definierte sich zunehmend als Verfolgungsopfer, das sich zur Wehr setzt. Halluzinationen hatte er verneint; ob er tatsächlich körperliche Beeinträchtigungserlebnisse gehabt hatte, war nicht zu klären.

Seine Überzeugungen haben in ihrer unkorrigierbaren Festigkeit und existenziellen Relevanz sicher Wahnqualität, sind aber nicht schizophren. Sie sind zwar abwegig, aber nicht im psychiatrischen Sinne bizarr, und sie haben nur einen begrenzten Bezug auf ihn selbst. Anders als ein Schizophrener beweist er seine wahnhaften Überzeugungen nicht aus eigenen Wahnwahrnehmungen, sondern durch den Verweis auf angebliche Experten im Internet. Da sind seine Theorien alle versammelt, und just so, wie er sie propagiert. Chemtrail-Gläubige gibt es seit 1996 im Internet; das Basismodell behauptet, bei den vermeintlich giftigen Kondensstreifen gehe es um eine gezielte Bevölkerungsreduktion (weil Wissenschaftler errechnet häten, die optimale Größe der Erdbevölkerung betrage 500 Millionen) oder auch um militärische Zwecke. Von Herrn A. werden Chemtrail und Impfgegnerschaft verschmolzen; das Impfen dient nun der bewussten Zerstörung der Menschen.

Der Wunderkasten der Physik und der digitalen und elektromagnetischen Wunderwaffen erlaubt viele Fantasien technischer Machbarkeit, als sei alles möglich, jede beliebige Miniaturisierung von Microchips auf Nanoebene, sodass sie per Injektion verabreicht werden können, oder auch die leiterfreie Übertragung großer Energiemengen. Warum nicht - je weniger Ahnung man hat, desto mehr hält man für möglich. In diesem Wunderland kann man dann beliebige Kausalverknüpfungen herstellen: Das autonome Fahren wird gefördert, weil große Teile der Bevölkerung umgebracht werden sollen.

Der schizophrene Wahn dient der vorläufigen Deutung und intrapsychischen Reparatur einer völlig aus den Fugen geratenen Wirklichkeit, die ihre natürliche Selbstverständlichkeit (Blankenburg 2012) verloren hat, in der nichts mehr stimmt, alles eine ganz andere Bedeutung haben kann und es keinerlei Zufall mehr gibt. Der schizophrene Wahn ist kein unverbrüchlicher fester Glaube, sondern er ist ein zweifelsfreies Wissen. So wie ich jetzt weiß und nicht nur glaube, dass ich in meinem Arbeitszimmer sitze, so ist der Schizophrene außerstande, sein psychotisches Erleben zu bezweifeln. Beweisend für die Schizophreniediagnose sind 
Wahnwahrnehmungen, also dass einer ganz gewöhnlichen Szene mit fragloser Gewissheit mit aufdringlichem Eigenbezug eine abwegige Bedeutung entnommen wird. Schneider (1976) benannte weitere Erstrangsymptome wie Gedankenentzug, Gedankenausbreitung oder kommentierende Stimmen. Wahneinfälle oder Wahnvorstellungen, sagte Schneider, gibt es reichlich und belegen noch keine Schizophrenie, sie sind Symptome zweiten Ranges.

Wahn im Rahmen der wahnhaften Störung erscheint in anderer Gestalt und Funktion: als das stabile, in Ideologien und konkrete Theorien gefasste Ressentiment gegen eine abweisende, kränkende, mit Lug und Trug arbeitende nahe und ferne soziale Umgebung und Obrigkeit. Der Betreffende sieht sich als Opfer, aber auch als tapferen Kämpfer, der sich nicht vor dem Unglück in der Welt verschließt und bereit ist, Widerstand zu leisten. Es stellt sich hier die Frage, ob die beklagten finsteren Machenschaften tatsächlich sinnlich erlebt wurden oder nur als stetes Risiko verhandelt werden. Der eigene Leib ist natürlich ein riesiges Terrain, in dem alle möglichen kleinen und großen Missempfindungen sich einfinden; auch hier möchte man unterscheiden können, zwischen Hypochondrie und den bizarren, oft schwer in Worte zu fassenden koenästhetischen Körpermissempfindungen Schizophrener.

Aber auch der schizophrene Wahn bedient sich des vorhandenen Deutungsmaterials und interpretiert das eigene Erleben unter Zuhilfenahme physikalisch-technischen Wissens. Strahlen sind seit Jahrzehnten eine häufig verwendete Erklärung, schon vor den Atombombenexplosionen in Hiroshima und Nagasaki, und danach verstärkt, aber auch Gifte, Gase, eingeflößte Substanzen, alles, was meine natürliche Schutzhülle durchdringen, in meinen Körper und meinen Geist eindringen kann. In den letzten Jahren waren es dann viele, die glaubten, man habe ihnen bei Arztbesuchen oder im Schlaf einen Chip implantiert, jetzt sind wir also so weit, dass die Chips mittels Impfung in den Körper gelangen. Diagnostisch ein wichtiger Unterschied ist aber, ob man misstrauisch befürchtet, dass mit Impfungen Chips eingeschleust werden, mit denen man fremdgesteuert werden kann, oder ob man diese Fremdsteuerung schizophren unmittelbar erlebt.

Auch der schizophrene Wahn zeigt bisweilen eine Anlehnung an zeitgeistige Themen oder Narrative, ist aber im Grundmuster zumeist originär selbstgemacht. Tobias Rathjen, der Attentäter von Hanau (Kröber 2020), entwickelt in seinem „Manifest“ zunächst eine eigene schizophrene Erzählung seiner Geburt und geburtsnah beginnenden Genialität, und dass er sich genau erinnere (mit exakter Pseudoexaktheit), wie er „5 Tage, 8 Tage oder 12 Tage nach der Geburt" erwachend von einer Frauenstimme angesprochen wird, nicht der seiner Mutter, die in ihm einen bleibenden Eindruck hinterlassen habe und im Zusammenhang mit der späteren Überwachung stehe. Rathjen schildert retrospekti- ve Wahnwahrnehmungen. Anders als die Verschwörungstheoretiker weiß Rathjen bis zum Schluss nicht, wer ihn verfolgt, wer die Geheimorganisation ist, und was ,das Rätsel“ ist. Und er schildert im Tone leichter Verwunderung, aber auch mit dem Stolz des Erwählten Erstrangsymptome wie Gedankenausbreitung und Gedankenentzug sowie kommentierende Stimmen.

Rathjen hat sich zumindest in seinen letzten Krankheitsund Lebensmonaten bei Narrativen bedient, die im Internet zu bekommen sind. Das Bundeskriminalamt hat seine Computer durchsucht und keinerlei Hinweise darauf gefunden, dass Rathjen sich für rechtsradikales oder rassistisches Material oder zumindest für politische Parteien interessiert hat. Aber seine Videobotschaft, die er wenige Tage vor der Tat an alle Amerikaner richtete, ist eine Kurzfassung der von „QAnon“ verbreiteten Legende, wonach Banker, Politiker und andere Mächtige massenhaft Kinder entführen und in unterirdischen Anlagen gefangen halten, wo sie gequält werden, um sie dann zu töten und ihnen Adrenochrom zu entnehmen. Adrenochrom wird für eine Anti-Aging-Substanz gehalten, mit der sich die global Mächtigen jung erhalten. Ursprünglich erklärte QAnon Barack Obama, Hillary Clinton und George Soros zu Zentralfiguren dieses Verbrechens, und Donald Trump galt seinen Anhängern als der Retter. Rathjen scheint spät auf diese Geschichte gestoßen zu sein; sie verschmilzt mit seinem Kampf gegen die „Geheimorganisation“, „die sich in die Gehirne der Menschen einklinkt und dort bestimmte Dinge abgreift, um dann das Weltgeschehen zu steuern".

Rathjen hat sein Manifest erst in den letzten Monaten vor der Tat vom 19.02.2020 um das Szenario der Menschheitsvernichtung ergänzt; der Bundesanwaltschaft hatte er es im November 2019 noch ohne dieses Abschlusskapitel vorgelegt. In ihm finden sich Denkfiguren aus den erfolgreichen Filmen der Reihe Marvel's Avengers (Marvel Studios, Burbank, CA, USA). Da ist zum einen der eigentümliche Gedanke der Halbierung der Weltbevölkerung, ein Motiv aus Infinity Wars. In dessen Nachfolgefilm, Avengers: Endgame, ist die Hälfte der Bevölkerung vernichtet, die Helden entwickeln aber den Gedanken und die Methode, um in die Vergangenheit zurückzukehren. Rathjen beendete sein Manifest mit dem Projekt, in einer Zeitschleife vor den Beginn der Menschen zurückzukehren und eine Menschheit ohne Leid ganz von Neuem aufzubauen. Denn man könne nicht „das millionenfache Leid, das Menschen erlitten haben, so stehen lassen“"

Avengers: Endgame kam im April 2019 in die Kinos, war weltweit der erfolgreichste Film des Jahres, ab 23.08.2019 digital erhältlich und ab 05.09.2019 als DVD und Blue-Ray. Es ist sehr wahrscheinlich, dass Rathjen den Film gesehen hat; er war auf Hollywood fixiert, weil er wähnte, dass dort seine Gedanken in Filme umgesetzt werden. 
Rathjen ist nicht durch Rechtsextremisten, aber auch nicht durch Marvel's Avengers und andere Science-Fiction-Filme zum finalen Amoklauf verleitet worden. Aber die katastrophische Grundstimmung und das heldenhafte Selbstopfer in Endgame korrespondierten sicher gut mit seinem Erleben. Im Juli 2019 hatte er einen Privatdetektiv aufgesucht in der Hoffnung, dieser könne Kontakt zum Geheimdienst und so zu Frau Merkel herstellen, von der er glaubte, sie wisse über diese Dinge Bescheid. Rathjen, im Anzug, habe äußerlich unauffällig gewirkt und sich ganz adäquat benommen. Aber schon nach seinen ersten Sätzen sei klar gewesen, dass das, was er vorträgt, völlig verrückt ist; dass jeder andere das so sehen muss, habe Rathjen aber gar nicht gespürt.

Nachdem seine Anzeigen vom November 2019 bei Staatsanwaltschaft und Bundesanwaltschaft ohne Resonanz blieben, scheint sein katastrophisches Erleben schließlich in eine Kapitulation vor der Übermacht der „,Geheimorganisation“ geführt zu haben. Sein „Endgame“ wurde kriegerisch umgesetzt im erneuten verstärkten Waffentraining und dann der Tötung fremder, dunkelhaariger Menschen, die für ihn wie fast alle Anderen minderwertig waren, in der Tötung der Mutter und seiner selbst. Bei der Opferwahl spielte neben pragmatischen Gründen sicherlich auch die Verachtung für Ausländer eine Rolle, von hier bis zu den Philippinen, ungeachtet ihrer „Rasse“, wenn es die denn gibt.

Um Katastrophen wie in Hanau zu verhindern, helfen keine neuen Antirassismusbeauftragten, sondern zupackende Hilfe, wenn sich bedroht fühlende Wahnkranke darum ersuchen, und Kontrolle ihres Zugangs zu Waffen. Erforderlich sind Standardregelungen für Polizeidienststellen und Staatsanwaltschaften, wie sie mit solchen Situationen aktiv umgehen. Psychiatrie hilft in solchen Fällen, nicht politpädagogische Belehrung.

Interessenkonflikt H.-L. Kröber gibt an, dass kein Interessenkonflikt besteht.

\section{Literatur}

Blankenburg W (2012) Der Verlust der natürlichen Selbstverständlichkeit. Ein Beitrag zur Psychopathologie symptomarmer Schizophrenien. Parodos, Berlin (Erstveröff. 1971)

Kröber H-L (2020) Blitzlicht: Hanau - ein schizophrener Gesinnungstäter? Forens Psychiatr Psychol Kriminol 14:220-222

Schneider K (1976) Klinische Psychopathologie, 11. Aufl. Thieme, Stuttgart (Erstveröff. 1946)

Witter H (1972) Wahn. In: Göppinger H, Witter H (Hrsg) Handbuch der forensischen Psychiatrie I. Springer, Berlin Heidelberg New York, S 470-475 